\title{
1 Efficient production of an avian adeno-associated virus vector using \\ 2 insect cell/baculovirus expression system
}

3 Anping Wang, Yongjuan Wang, Shuang Wu, Weiyong Zuo, Changming Guo, Weiming Hong,

4 Shanyuan Zhu*

5

Jiangsu Agri-animal Husbandry Vocational College, Jiangsu Key Laboratory for High-Tech Research and Development of Veterinary Biopharmaceuticals, Taizhou, 225300, China

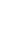

\section{ABSTRACT}

Recombinant avian adeno-associated virus (rAAAV) is a promising gene transfer vector for avian cells. Although rAAAV can be produced by co-transfection of HEK293 cells with three plasmids, both scalability and productivity of the transient transfection method can not meet the demand for large-scale in vivo experiments. In this study, a scalable rAAAV production method was established by using insect cell/baculovirus expression system. Three recombinant baculoviruses, namely BacARep, BacAVP and BacAGFP, were generated by transfection of Sf9 cells with the three plasmids expressing AAAV Rep genes, modified VP gene or the inverted terminal repeats-flanked green fluorescent protein (GFP) gene. After demonstration of the correct expression of AAAV genes, rAAAV-GFP was produced by triple infection of insect cells or triple transfection of HEK293 cells for comparison purpose. Electron microscopy revealed the formation of typical AAAV particles in the insect cells. Western blotting showed the correct assembly of rAAAV particles with a VP protein ratio similar to that of AAAV. Quantitative PCR showed that the insect cell-produced rAAAV yield was almost 25-fold higher than that produced by HEK293 cells. Fluorescent microscopy showed that the insect cell-produced rAAAV could transfer GFP reporter gene into two avian cell types with similar transfer efficiency to 

system could be used for scalable production of rAAAV, and the viral vector produced could be used as

25 the gene transfer vehicle for avian cells.

26 Key words: Insect cell/baculovirus expression system; Recombinant avian adeno-associated virus 


\section{Introduction}

Adeno-associated virus (AAV) vectors are popular gene transfer vehicles for gene therapy and vaccine development due to their quasi-absence of pathogenicity, excellent safety and long-term gene expression in a wide range of tissues (Warrington and Herzog, 2006). The traditional methods for producing recombinant AAV (rAAV) employ co-transfection of human embryonic kidney (HEK) 293 cells with the rAAV vector and helper vector, followed by infection with the helper adenovirus to induce the replication of rAAV (Samulski et al., 1987). The main drawback of this approach is the co-production of rAAV and helper virus. This problem can be overcome by replacing the helper virus with the plasmid containing necessary functions for the replication of rAAV (Matsushita et al., 1998; Salvetti et al., 1998; Xiao et al., 1998). However, the transient transfection method has limited scalability and relatively high incidence of replication competent AAV production (Galibert and Merten, 2011).

Three scalable rAAV production systems have been developed, including the use of AAV packaging and/or producer cell lines (Farson et al. 2004), use of recombinant herpes simplex virus vectors (Booth et al. 2004), and use of insect cell/baculovirus expression system (Galibert and Merten, 2011). The third system employs triple infection of insect cells with Rep-baculovirus, VP-baculovirus and AAV ITR vector genome baculovirus (Urabe et al., 2002). This system has the volumetric production yield in the range of $1.1 \times 10^{14} \mathrm{vg} / \mathrm{L}$, and thus is gaining in popularity in the production of viral vectors or virus-like particles (Aucoin et al., 2006; Galibert and Merten, 2011).

Avian adeno-associated virus (AAAV) was first isolated as a contamination virus from the Olson strain of quail bronchitis virus, an avian adenovirus (Yates et al., 1973). Sequence analysis shows that 
AAAV shares the similar genome structure with AAV (Bossis et al., 2003; Estevez et al., 2004; Wang et al., 2011), indicating its potential use as the gene transfer vector for avian cells. Like other AAVs, recombinant AAAV (rAAAV) can be produced by co-transfection of HEK 293 cells with the AAAV vector containing gene of interest, helper vector containing rep and cap genes, and helper plasmid containing necessary functions for the replication of rAAAV (Bossis et al., 2003; Estevez et al., 2004; Wang et al., 2007). However, both scalability and productivity of the transient transfection method are limited, which restrict the in vivo use of rAAAV vectors. To overcome these limitations, in this study an efficient platform for rAAAV production was generated by using insect cell/baculovirus expression system.

\section{Materials and methods}

\subsection{Cells and plasmid vectors}

Spodoptera frugiperda Sf9 cells (Invitrogen, Germany) were grown at $27^{\circ} \mathrm{C}$ in Sf-900 Iserum-free medium (Gibco, USA). Human embryonic kidney (HEK) 293 cells (ATCC CRL-1573, USA), chicken embryonic fibroblast DF1 cells (ATCC CRL-12203) and chicken embryonic liver (CEL) cells (ATCC CRL-2117) were grown in Dulbecco's modified Eagle's medium (DMEM, Gibco) supplemented with $10 \%$ fetal calf serum (FCS). Plasmid vector pCR-AAAV containing the complete genome of AAAV was kindly provided by Dr. J. Y. Wang at the College of Veterinary Medicine, Yangzhou University.

Green fluorescent protein (GFP) reporter gene-containing AAAV transfer vector pAITR-GFP, AAAV packaging vector pcDNA-ARC and adenovirus helper vector pHelper were provided by Dr. H. Sun at the College of Veterinary Medicine, Yangzhou University. 
AAAV VP (AVP) gene was amplified by polymerase chain reaction (PCR) from pCR-AAAV $\begin{array}{lllll}\text { vector (Wang } & \text { et } & \text { al., } & \text { 2011) } & \text { using }\end{array}$

5'-TTACAGCGGTTTGGTGAGGTAACGGGTACCG-3'. The mutated bases relative to the wild-type sequence are italicized. To express the three VP polypeptides from a single transcript in a correct ratio (1:1:10) without RNA splicing, the ATG initiation codon of VP1 was mutated at position 2 to ACG to reduce its translation efficiency. The downstream out-of frame ATG codon with diminished translation of VP2 and VP3 was modified at position 18 to create an ACG triplet. The splice acceptor site downstream of the VP1 initiation codon was mutated at position 24 to prevent the pre-mRNA splicing (Urabe et al., 2002). The PCR product was subcloned into baculovirus vector pFastBac1 (Invitrogen) as a NotI/HindIII segment, and the recombinant vector was called pFBAVP. AAAV Rep78 (ARep78) gene was amplified using primers 5'-ATGAGGTCGTACTACGAGGTC-3' and 5'-TCAGCCGCAGCGTTGACTCCC-3'. The PCR product was subcloned into pFastBacDual vector (Invitrogen) as a NheI segment, and the recombinant vector was called pFBARep78. AAAV Rep52 (ARep52) gene was amplified using primers 5'-ATGGAGCTCGTGGATTGGCTC-3' and 5'-TCAGCCGCAGCGTTGACTCCC-3'. The PCR product was cloned into pFBARep78 vector as a SalI/NotI segment, and the new recombinant vector was called pFBARep. The GFP expression cassette was amplified from pEGFP-N1 vector (Clontech, USA) using primers 5'-TAGTTATTAATAGTAATC-3' and 5'-CTTAAGATACATTGATG-3'. The PCR product was cloned into pCR-AAAV vector after removing the sequences in between the two ITRs by PmlI/BsmBI digestion. The ITR-flanked GFP expression cassette was subcloned into pFastBacDual vector as an 
EcoRI segment, and the resultant rAAAV vector was called pFBAGFP. The recombinant vectors pFBAVP, pFBARep and pFBAGFP were used to produce recombinant baculoviruses, namely BacAVP, BacARep and BacAGFP, by following the manufacturer's instruction of Bac-to-Bac Baculovirus Expression System (Invitrogen).

\subsection{Detection of AAAV Rep and VP expression in insect cells by immunofluorescence}

To confirm the correct expression of AAAV rep and cap genes in insect cells, Sf9 cells were seeded on 24-well plates $\left(6 \times 10^{5} /\right.$ well $)$, incubated for $1 \mathrm{~h}$, and infected with BacARep or BacAVP (MOI 1). On day 3 post-infection, the cells were fixed for $20 \mathrm{~min}$ at room temperature with $4 \%$ paraformaldehyde and blocked for $60 \mathrm{~min}$ with 5\% BSA in PBS. The standard indirect immunofluorescence was performed using mouse anti-ARep (1:100) or anti-AVP (1:200) serum, which was prepared by immunizing mice with E. coli-expressed AAAV Rep or Cap protein as described previously ( $\mathrm{Li}$ et al., 2013). After incubation with FITC-labeled goat anti-mouse IgG (1:500, Jackson ImmunoResearch, USA), the cell cultures were observed under microscope for fluorescently positive cells.

\subsection{Detection of AAAV Rep and VP expression by Western blotting}

Sf9 cells were infected with BacARep or BacAVP as described. The cell extracts were run on 12\% SDS-PAGE and the separated proteins were transferred onto PVDF membranes (Millipore, USA) using Semi-Dry Transfer Unit (TE70X, Hoefer, USA). The membranes were blocked overnight at $4^{\circ} \mathrm{C}$ with $10 \%$ skimmed milk in PBST, and incubated for $2 \mathrm{~h}$ at room temperature with mouse anti-ARep (1:100) or anti-AVP (1:200). After three time washing in PBST, the membranes were incubated for $1 \mathrm{~h}$ with HRP-labeled goat anti-mouse IgG (1:2000, Jackson Immuno Research), and the signals were 
developed with 3, 3'-diaminobenzidine substrate.

\subsection{AAAV vector production and purification}

To produce a rAAAV vector using the insect cell/baculovirus expression system, Sf9 cells were grown to $2 \times 10^{6} / \mathrm{ml}$ in suspension, and infected with BacAGFP, BacAVP and BacARep (MOI 5 each).

For the comparison purpose, HEK 293 cells were grown to the same density $\left(2 \times 10^{6} / \mathrm{ml}\right)$ and transfected with pAITR-GFP, pcDNA-ARC and pHelper as described previously (Wang et al., 2007).

Three days after infection or transfection, the Sf9 cells were collected by centrifugation, and HEK 293 cells were submitted to 3 cycles of freeze-thawing before centrifugation. The resultant rAAAV vector, namely rAAAV-GFP, was purified by PEG precipitation method as described previously (Matsushita et al., 1998). The purified virus was titrated in triplicates by real-time PCR using GFP-specific primers addition, the rAAAV-GFP was also titrated on DF1 cells as transduction units (TU)/ml as described previously (Wang et al., 2007).

\subsection{Identification of $r A A A V$ vector}

To identify rAAAV-GFP morphologically, Sf9 cells were infected with BacAGFP, BacAVP and BacARep as described, and the cells were harvested by centrifugation for the ultrathin section preparation and rAAAV purification on day 3 post-infection. The intracellular rAAAV-GFP particles were observed under transmission electron microscope (UC6, Leica, Germany). The purified rAAAV-GFP was strained negatively for 1 min with $2 \%$ phosphotumgstic acid (pH7.4) and observed 
under transmission electron microscope (Tecnai 12, FEI, USA). To identify rAAAV-GFP immunologically, the purified viral particles were analyzed by Western blotting using mouse anti-AVP serum as described.

\section{7. rAAAV-mediated GFP expression in avian cells}

To test the gene transfer efficiency of rAAAV-GFP produced in insect cells, DF1 cells and CEL cells were seeded on 24 -well plates $\left(1 \times 10^{5} /\right.$ well $)$ and infected with serial dilutions of rAAAV-GFP after incubation for $24 \mathrm{~h}$. The cell cultures were observed directly under microscope for fluorescent-positive cells $24 \mathrm{~h}$ post-infection.

\section{Results}

\subsection{Three rBacs for producing $r A A A V$ were generated}

The PCR products of ARep78 and ARep52 genes were subcloned into baculovirus expression vector pFastBacDual, in which the expression of two rep genes were under the control of p10 and ph promoters, respectively (Fig. 1). The recombinant vector pFBARep was transfected into Sf9 cells, and the resultant BacARep was used to infect Sf9 cells. By using anti-ARep serum as the first antibody, immunofluorescence showed that the two Rep genes were expressed efficiently in BacARep-infected insect cells (Fig. 2A). Furthermore, Western blotting detected the expected 80- and 55-kDa protein bands in BacARep-infected cells, but not in mock-infected cells (Fig. 2B).

To express the three AVP in insect cells and in a correct ratio, the strategy similar to BacVP vector construction (Urabe et al., 2002) was used to design BacAVP vector, including mutation of the ATG codon of VP1 into ACG to reduce its translation efficiency, mutation of the ATG codon with diminished translation of VP2 and VP3 to create an ACG triplet, and destroy of the splice acceptor site 
downstream of VP1 initiation codon to prevent the pre-mRNA splicing. The PCR product of AVP gene was cloned into baculovirus expression vector, and the resultant pFBAVP vector was transfected into Sf9 cells to produce BacAVP. By using anti-AVP serum as the first antibody, immunofluorescence showed that the AVP gene was expressed correctly in BacAVP-infected insect cells (Fig. 2A). Moreover, Western blotting analysis showed that the three VP proteins were expressed in the expected ratio of about 1:1:10 with the expected molecular weights of 83, 66 and $60 \mathrm{kDa}$, respectively (Fig. 2C).

Both Rep and Cap genes of AAAV were removed from pCR-AAAV vector by restriction digestion, and the resultant ITR-containing vector was ligated to the GFP expression cassette amplified from pEGFP-N1 vector. The recombinant vector pFBAGFP was transfected into Sf9 cells and the resultant BacAGFP was harvested for infection of Sf9 cells. Fluorescent microscopy showed efficient expression of GFP in the rBac-infected cells (data not shown).

\subsection{Correct $r A A A V$ is produced by triple infection of insect cells}

To identify rAAAV-GFP produced in insect cells, Sf9 cells were infected with BacAGFP, BacAVP and BacARep, and the infected cells were collected for ultrathin section preparation and rAAAV-GFP purification on day 3 post-infection. Transmission electron microscopy revealed AAV-like viral particles clustered in the nucleus of Sf9 cells (Fig. 3A). Under electron microscope, the purified viral particles had an icosahedral structure with a diameter of about $22 \mathrm{~nm}$, which was morphologically similar to other parvovirus particles (Fig. 3B). In addition, three expected VP protein bands were detected by Western blotting in rAAAV-GFP-infected cells, but not in the mock-infected cells (Fig. $3 C)$. 
175

To compare the volumetric rAAAV yields of the two different production systems, the same number $\left(2 \times 10^{8}\right)$ of Sf9 or HEK 293 cells was used to produce rAAAV-GFP by triple infection or triple transfection. The quantitative PCR analysis showed that up to 28, 000 rAAAV particles could be produced by each Sf9 cell, which was almost 25 -fold more than that produced by each HEK 293 cell (Table1).

\subsection{Insect and mammalian cell-produced rAAAV has similar gene transfer efficiencies for avian cells}

To compare the gene transfer efficiencies of rAAAV produced by two different production systems, DF1 and CEL cells were infected with the same dose of rAAAV-GFP produced by Sf9 or HEK 293 cells. Fluorescent microscopy showed that the two types of avian cells could be transduced by rAAAV-GFP produced by Sf9 cells or HEK 293 cells with similar transduction efficiencies (Fig. 4).

\section{Discussion}

AAAV can be used as the efficient gene transfer vectors for avian cells. Although rAAAV vectors have been produced successfully by triple transfection of HEK 293 cells (Perozo et al., 2008; Wang et al., 2008; Wang et al., 2009), both scalability and viral titer of the transient transfection method can not meet the demand for in vivo experiments. Among the scalable rAAV production systems developed, insect cell/baculovirus expression system is the most promising platform for large-scale production of rAAV vectors (Galibert and Merten, 2011). This warranted us to explore the feasibility of insect cell/baculovirus expression system for scalable production of rAAAV vectors. To this end, we generated three recombinant baculoviruses containing AAAV Rep genes, VP gene or ITR-flanked GFP reporter gene. Among these recombinant baculoviruses, the proportional expression of three VP 
structural proteins is essential for the assembly of rAAAV particles in insect cells. For example, co-infection of insect cells with three baculoviruses expressing AAV VP1, VP2 and VP3 individually leads to the formation of protein aggregates rather than correct rAAV particles (Urabe et al., 2002). The main reason is due to the presence of introns in the VP gene which can not be spliced out from the pre-mRNA by insect cells (Chen, 2008). This problem has been solved successfully by expressing the three VP proteins with mutated initiation codons in the absence of RNA splicing (Urabe et al., 2002). Therefore, in this study the ATG initiation codon of AAAV VP1 was mutated to ACG to reduce the translation efficiency. The downstream out-of frame ATG codon with diminished translation of VP2 and VP3 was also modified to create an ACG triplet. In addition, the splice acceptor site downstream of the VP1 initiation codon was mutated to prevent the pre-mRNA splicing. Western blotting analysis of BacAVP-infected Sf9 cells showed that the three AAAV VP proteins were expressed with the expected ratio, indicating the usability of the rBac for producing rAAAV in insect cells.

To demonstrate the feasibility of above strategy for scalable production of rAAAV vectors, insect cells were co-infected with BacARep, BacAVP and BacAGFP, and the infected cells were harvested for ultrathin section preparation and rAAAV purification. Electron microscopy showed the presence of typical parvovirus particles clustered in the nucleus of rBac-infected cells. The rAAAV particles could be purified to a relatively high purity by PEG precipitation. Western blotting analysis showed that the purified viral particles had a VP protein ratio similar to that of AAAV. These data suggest the efficient expression of AAAV proteins and correct assembly of the viral particles in the rBac-infected insect cells without the helper viruses. To compare the production performance of triple infection method with that of triple transfection, the same numbers of insect cells and HEK293 cells were used to produce GFP gene-containing rAAAV vector. Quantitative PCR showed that each insect cell could produce 28, 
000 rAAAV particles, which was almost 25-fold more than that (1100) of each HEK293 cell. Up to 5.6 $\times 10^{12}$ rAAAV could be obtained from $2 \times 10^{8}$ insect cells, indicating the relatively high scalability of insect cell/baculovirus expression system for producing rAAAV vectors.

Another important issue to be addressed was whether the rAAAV vector produced in insect cells could transfer gene of interest into avian cells. To answer this question, DF1 cells and CEL cells were transduced with the BacAGFP produced in insect cells or HEK293 cells under the same conditions. Fluorescent microscopy revealed the similar positive cell numbers in the two types of avian cell cultures after transduction with the rAAAV produced using two different methods. The expression of GFP reporter gene lasted for at least 2 weeks. These data suggest the potential use of insect cell-produced rAAAV vectors as efficient gene transfer vehicle for avian cells.

In conclusion, the insect cell/baculovirus expression system established in this study could become a scalable platform for producing rAAAV vectors, and the rAAAV produced could be used as efficient gene transfer vector for avian cells.

\section{Acknowledgements}

This work was supported by grants from the Chinese National Science Foundation (31302096), the Natural Science Foundation of Jiangsu Province, China (BK2011536), the Science and Technology Project of Jiangsu Province, China (BE2013415), the Six Talent Peaks Project of Jiangsu Province, China (NY-009). We thank Dr. H. Sun at the College of Veterinary Medicine, Yangzhou University for polishing the manuscript. All authors do not have conflict of interest to declare for this study.

\section{References}

Aucoin, M.G., Perrier, M., Kamen, A.A., 2006. Production of adeno-Associated viral vectors in insect 
cells using triple infection: optimization of baculovirus concentration ratios. Biotechnol. Bioeng. $95(6), 1081-1092$.

Booth, M.J., Mistry, A., Li, X., Thrasher, A., Coffin, R.S., 2004. Transfection-free and scalable recombinant AAV vector production using HSV/AAV hybrids. Gene Ther. 11, 829-837.

Bossis, I., Chiorini, J.A., 2003. Cloning of an avian adeno-associated virus (AAAV) and generation of recombinant AAAV particles. J. Virol. 77 (22), 6799-6805.

Matsushita, T., Elliger, S., Eliger, C., Podsakoff, G., Villarreal, L., Kurtzman, G.J., Iwaki, Y., Colosi, P., 
Perozo, F., Villegas, P., Estevez C., Alvarado, I. R., Purvis, L. B., Williams, S., 2008. Protection against infectious Bursal disease virulent challenge conferred by a recombinant avian adeno-associated virus vaccine. Avian Dis. 52(2), 315-319.

Perozo, F., Villegas, P., Estevez C., Alvarado, I. R., Purvis, L. B., Saume, E., 2008. Avian

Salvetti, A., Orève, S., Chadeuf, G., Favre, D., Cherel, Y., Champion-Arnaud, P., David-Ameline, J.,

Urabe, M., Ding, C.T., Kotin, R.M., 2002. Insect cells as a factory to produce adeno-associated virus type 2 vectors. Hum. Gene Ther. 13, 1935-1943.

Wang, A.P., Sun, H.C., Wang, J.Y., Wang, Y.J., Yuan, W.F., 2007. Construction and identification of recombinant avian adeno-associated virus expressing GFP reporter gene. Chin. J. Virol. 23(4), 391-394. (in Chinese, abstract in English)

Wang, A.P., Sun, H.C., Wang, Y.J., Wang, J.Y., Yuan, W.F., 2008. Recombinant avian adeno-associated virus-mediated oviduct-specific expression of recombinant human tissue kallikrein. Poultry Sci. 87, 

Virol. 156, 71-77.

284 Wang, Y.J., Sun, H.C., Shen, P.P., Zhang, X.Y., Xia, X.L., 2009. Effective inhibition of infectious bursal disease virus replication by recombinant avian adeno-associated virus-delivered microRNAs. J. Gen. Virol. 90, 1417-1422

Warrington, K.H., Herzog, R.W., 2006. Treatment of human disease by adeno-associated viral gene transfer. Hum. Genet. 119, 571-603.

Xiao, X., Li, J., Samulski, R.J., 1998. Production of high-titer recombinant adeno-associated virus vectors in the absence of helper adenovirus. J. Virol. 72, 2224-2323.

Yates, V.J., el-Mishad, A.M., McCormick, K.J., Trentin, J.J., 1973. Isolation and characterization of an avian adeno-associated virus. Infec. Immun. 7, 973-980. 


\section{Figure captions}

Fig.1. The Schematic structures of recombinant baculovirus vectors used for producing rAAAV.

pFBARep harbors Rep78 and Rep52 expression cassettes. Rep78 is expressed by the p10 promoter and Rep52 is expressed by the ph promoter. pFBAVP expresses capsid proteins VP1, VP2, and VP3 under the control of ph promoter. The ATG codon of VP1 is mutated to ACG, enabling the expression of all three VP proteins from one transcript. pFBAGFP carries a GFP expression cassette flanked by AAAV ITRs. GFP is expressed by the CMV promoter.

Fig.2. Detection of AAAV Rep and Cap gene expression in the rBac-infected insect cells.

(A) Indirect immunofluorescence assay of AAAV Rep and Cap gene expression in rBac-infected Sf9 cells using mouse anti-ARep or anti-AVP serum. (B) Western blotting assay of AAAV Rep gene expression in rBacARep-infected Sf9 cells using mouse anti-ARep. (C) Western blotting assay of AAAV Cap gene expression in rBacAVP-infected Sf9 cells using mouse anti-AVP. Lane M, prestained protein molecular weight marker; Lane 1, Sf9 cells not infected with recombinant baculovirus; Lane 2, Sf9 cells infected with recombinant baculovirus rBacARep or rBacAVP.

Fig.3. Identification of rAAAV-GFP produced in rBac-infected insect cells.

(A) Ultrathin section electron microscopy of Sf9 cells infected with the three recombinant baculoviruses, rBacAGFP, rBacAVP, and rBacARep. Original magnification: ×21 000. Bar: 100 nm. (B) Electron microscopy of purified rAAAV strained with $2 \%$ phosphotumgstic acid. Original magnification: $\times 97$ 000. Bar: $100 \mathrm{~nm}$. (C) Western blotting analysis of purified rAAAV. Lane 1, Sf9 cells not infected with recombinant baculovirus; Lane 2, purified rAAAV particles by PEG precipitation.

Fig.4. Detection of rAAAV-mediated GFP reporter gene expression in avian cells. 
293 cells and observed directly under microscope $24 \mathrm{~h}$ post-infection. 

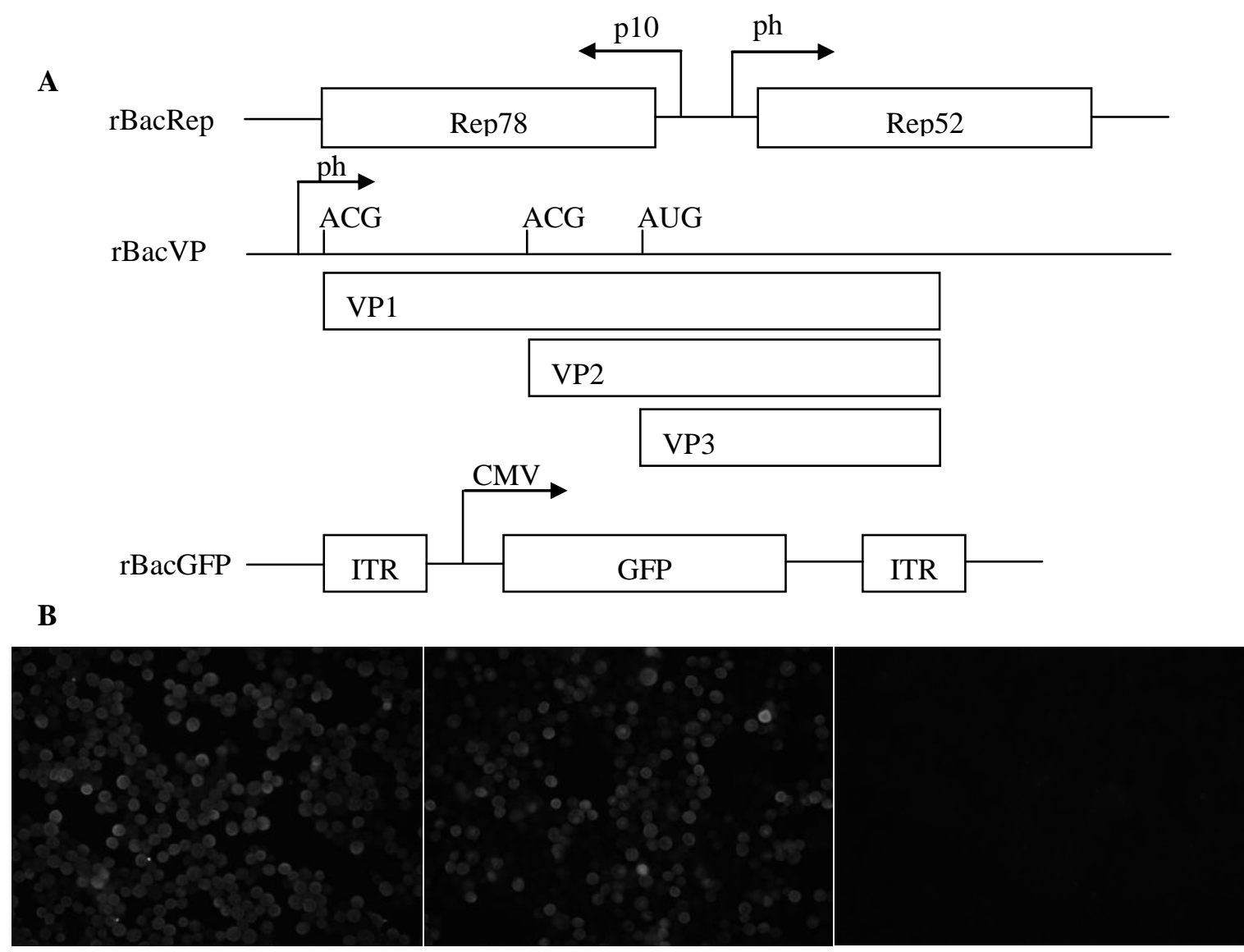

rBacRep+anti-Rep

rBacVP+anti-VP

Sf9+native mouse serum
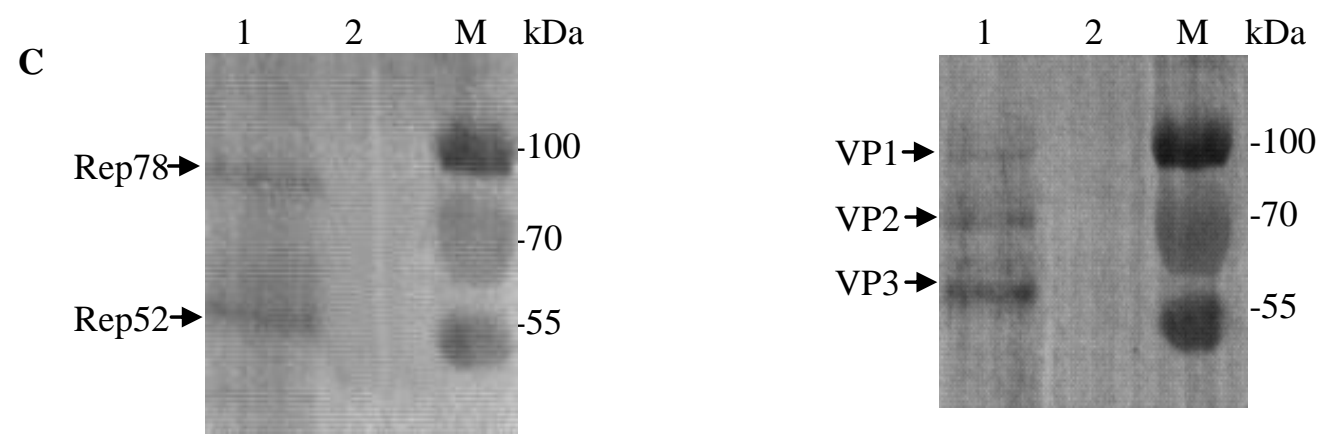

Fig.1. (A) Transcription representations of the recombinant baculoviruses. rBacRep harbors Rep78 and Rep52 expression cassettes. Rep78 is expressed by the p10 promoter and Rep52 is expressed by the ph promoter. rBacVP expresses capsid proteins VP1, VP2, and VP3 under the control of ph promoter. The ATG codon of VP1 is mutated to ACG, enabling the expression of all three VP proteins from one transcript. rBacGFP carries a GFP expression cassette flanked by AAAV ITRs. GFP is expressed by the CMV promoter. (B) Indirect immunofluorescence assay of recombinant proteins expressing in Sf9 cells infected with recombinant baculovirus.

(C) Western blot analysis of recombinant proteins expressing in Sf9 cells infected with recombinant baculovirus. Lane 1 represents $\mathrm{Sf} 9$ cells infected with recombinant baculovirus rBacRep or rBacVP; Lane 2 represents Sf9 cells not infected with recombinant baculovirus. 
A

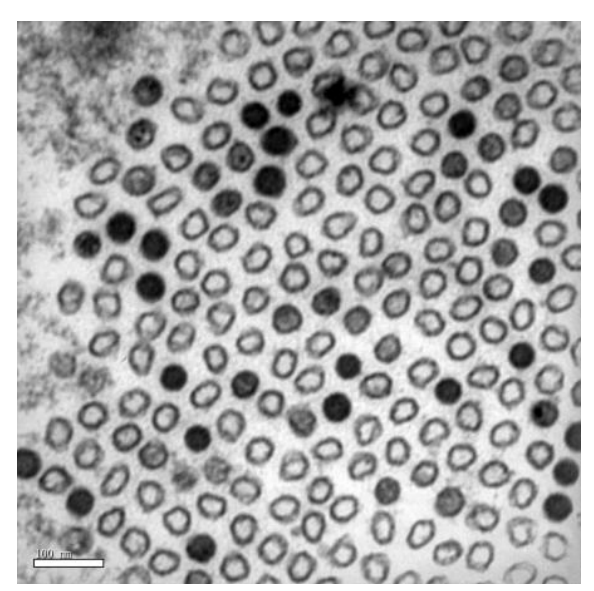

B

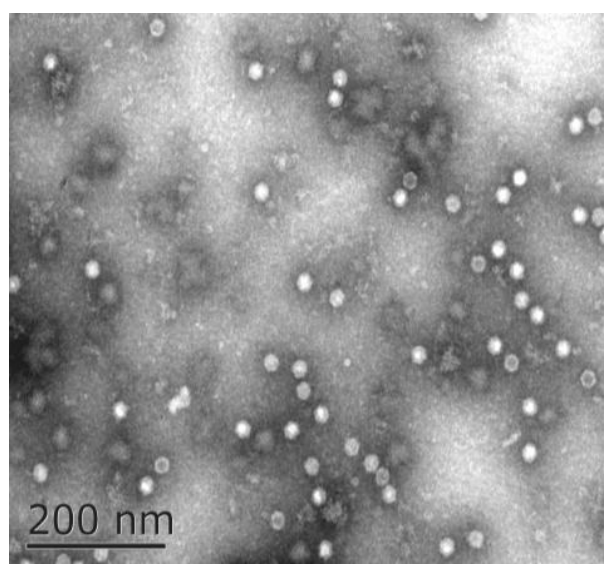

2

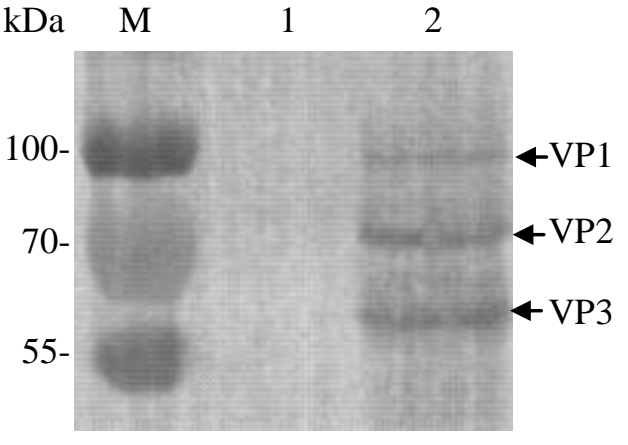

Fig.2. Identification of rAAAV produced in Sf9 cells. (A) Ultrathin section electron microscopy of Sf9 cells infected with the three recombinant baculoviruses, rBacGFP, rBacVP, and rBacRep. Original magnification: $\times 21000$. Bar: $100 \mathrm{~nm}$. (B) Electron microscopy of purified rAAAV strained with $2 \%$ phosphotumgstic acid. Original magnification: $\times 59000$. Bar: $200 \mathrm{~nm} .(\mathrm{C})$ Western blotting analysis of purified rAAAV. Lane 1 represents Sf9 cells not infected with recombinant baculovirus; Lane 2 represents purified rAAAV. 
Table 1. Comparison of rAAAV yield between two methods

\begin{tabular}{cccc}
\hline & $\begin{array}{c}\text { Total yield } \\
\left(\times 10^{11} \mathrm{VG}\right)\end{array}$ & $\begin{array}{c}\text { Per cell } \\
\left(\times 10^{3} \mathrm{VG}\right)\end{array}$ & $\mathrm{VG}^{\mathrm{TT}}{ }^{\mathrm{a}}$ \\
\hline Sf9 cells $\left(2 \times 10^{8}\right)$ & $56 \pm 1.6$ & $28 \pm 0.8$ & $1562 \pm 358$ \\
293 cells $\left(2 \times 10^{8}\right)$ & $2.2 \pm 0.89$ & $1.1 \pm 0.45$ & $1936 \pm 517$ \\
\hline
\end{tabular}

${ }^{\mathrm{a}}$ Vector genomes per transducing unit. 


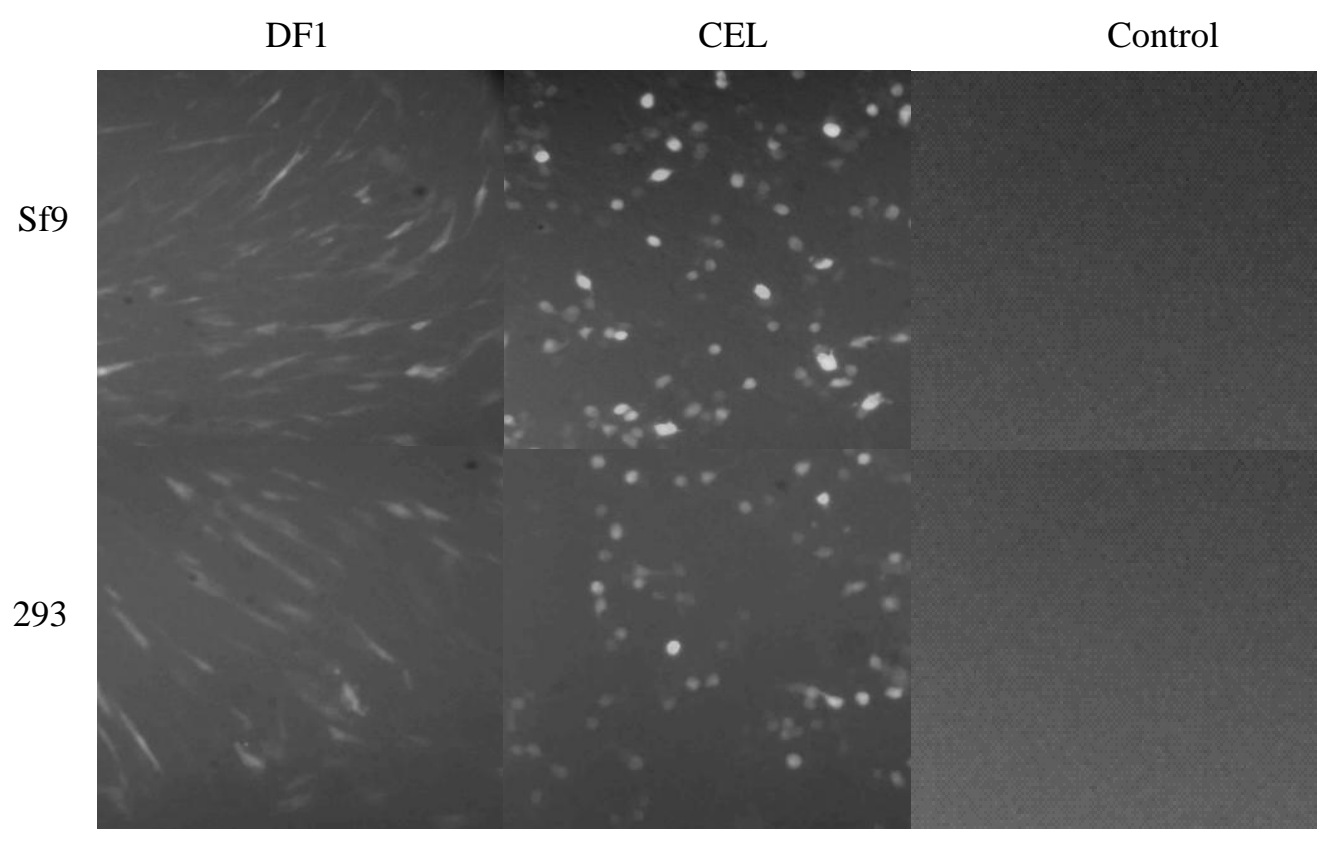

Fig.3. Expression of GFP in DF1 cells or CEL cells infected with rAAAV-GFP produced in Sf9 cells or 293 cells. 